\title{
Modular Rapidly Manufactured Small Satellite (MRMSS)
}

\author{
Greenfield T. Trinh ${ }^{1}$, Daniel Cellucci ${ }^{2}$, Will Langford ${ }^{3}$, \\ Stephen $\mathrm{Im}^{4}$, Ali Guarneros Luna ${ }^{5}$, Kenneth C. Cheung ${ }^{6}$ \\ NASA Ames Research Center, Mountain View, CA, 94035
}

The Modular Rapidly Manufactured Small Satellite (MRMSS) project applies modular building block system to space applications. The need to reduce mass for spaceflight applications and reuse resources is a critical technology for long duration space missions. Mass reduction and reuse of material will help bring down costs for spaceflight missions and open up more possibilities for exploration and research. The MRMSS project consists of two major components: A basic research component demonstrating electronic digital materials, and a technology demonstration applying the modular building block based systems concept to the CubeSat form factor. This paper describes the core technologies developed to enable the modular system as well the first flight demonstration on a sounding rocket.

\section{Introduction}

Digital materials are materials with "a discrete set of components, discrete number of allowable relative positions and orientations, and explicit control of the placement of individual components"[1] [2]. The motivation behind the MRMSS project's application to spacecraft structures stems from the need to reduce mass carried into orbit and reuse of the material once it has served its original purpose. Ongoing research at NASA Ames seeks ways to make spacecraft systems more versatile, and the CubeSat platform is a appropriately suited for demonstrating this concept due to its inherent modularity. By defining each side of the CubeSat as a single modular unit, we have developed a system that is scalable, modular, and modifiable. This enables the launch of densely flat-packed standard CubeSat face templates that can be deployed to structures that are as large as needed.

\footnotetext{
1 Mission Design Division, NASA Ames Research Center, AIAA Member

2 Department of Mechanical and Aerospace Engineering, Cornell University

3 Center for Bits and Atoms, Massachusetts Institute of Technology

${ }^{4}$ Department of Aerospace Engineering, San Jose State University

${ }^{5}$ Mission Design Division, NASA Ames Research Center

6 Office of the Center Chief Technologist, NASA Ames Research Center
} 
The MRMSS project has two major components: A basic research collaboration demonstrating electronic digital materials and a technology demonstration that is aimed at taking appropriate components of the research work and reducing it quickly to practice as a flight demonstration. For the technology demonstration effort, we show a near-term application of building block systems strategy to spacecraft systems. Small satellites are considered as a suitable platform both because of the shared goal of modularity and the ability to perform a technology demonstration relatively quickly.

\section{Technology Development}

The technology development of the MRMSS project is comprised of two main sections: modular architecture, and networking technologies. The modular architecture research work addresses the base spacecraft card system and how each module will connected to each other mechanically and electrically. The network technologies work investigates different wired and wireless technologies that is needed to enable communication between the modules.

\section{A. Modular Architecture}

Modular discrete material concepts stem from information theory and seek to achieve natural scalability in hardware systems. Discrete building blocks have the advantage of being replaceable, reconfigurable, and can be manufactured in high volume. Because of those advantages, modular architectures are suited for spaceflight applications. Long term space travel will need to maximize the utility of resources on hand and modular architectures will help enable that capability.

Modular architecture development for the MRMSS project uses each side of a CubeSat as a discrete unit. The geometry of the units and therefore the aggregate systems can depend on the application. For the MRMSS project, each unit is a PCB board that includes a microcontroller, a payload, and connectors. The board is based off an Arduino Pro Mini 3.3V [3]. Using the Arduino [4] platform, each board has the capability to integrate a payload using the serial communication pins. The boards will be able to communicate with neighboring units using the Asynchronous Packet Automata (APA)[5] protocols that will be discussed in the next section. Each board is powered by a $850 \mathrm{mAh}$ lithium polymer battery. The system is designed to accommodate Triangular Advanced Solar Cells (TASC) [6] cell solar panels. Using these boards as a base, each one can carry an instrument such as a radio, sensor, reaction wheel, or any other device. This design builds upon concepts from past projects such as NASA's M-PACE [7] and Cornell University's KickSat Project.[8] 


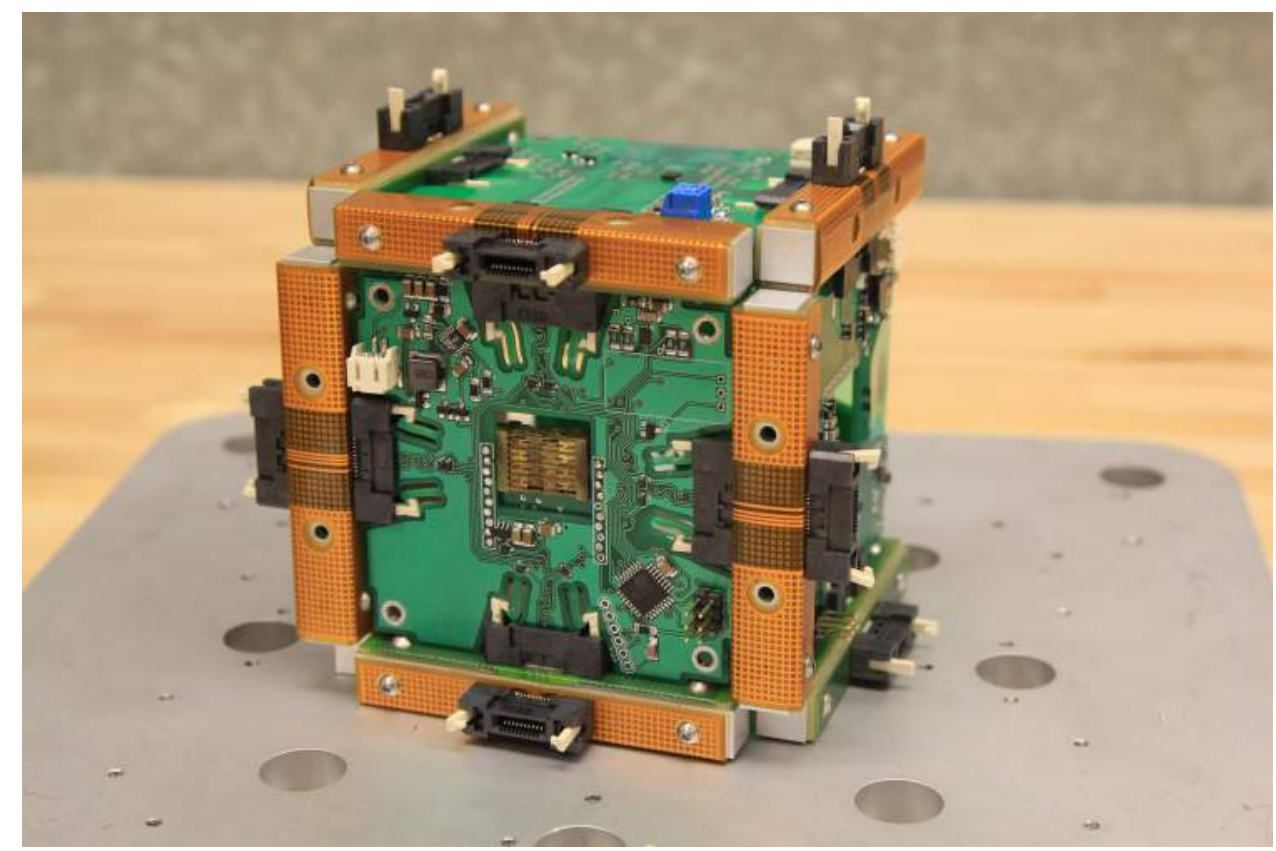

Fig. 1: Image of an assembled 1U MRMSS system with the MIT payload attached.

Each module is connected to neighboring sections using a "tie" consisting of an aluminum base structure with a flexible PCB connector wrapped around (as shown in Fig. 1). The tie structure allows for data and power transfer between each module.

\section{B. Networking Technologies}

One of the necessities that result from a modular architecture is communication between each module. Networks within the system will allow for each module to communicate with each other, with information such as health status. A mesh network topology is suited for this type of system because each module is connected in a way where information can be routed from one module to any other one in the system. Inter-structure connectivity is an important aspect of the modular architecture. We investigated wired and wireless solutions for this application. Ultimately, we decided to implement a wired network as the baseline for this project. Once the final system is built, we will implement a wireless network using the payload slots housing a XBee [9] module.

\section{Wired Network}

Two criteria motivated the selection of the wired networking algorithm for the MRMSS test suite: scalability and simplicity. Though the test apparatus consists of only four boards, the vision for the work is for a system composed of many hundreds of components connected in a cubic array through the aforementioned ties. For a fully connected system, this means that each of the microcontrollers 
present on the board must be able to communicate with 12 immediate neighbors and potentially hundreds of secondary neighbors. Additionally, the desire for low cost and the quasi-static physical topology meant that geometry-independent addressing schemes such as TCP/IP were not worth the additional processing overhead.

As a result, we selected a protocol developed by MITs Center for Bits and Atoms, called the Asynchronous Packet Automaton (APA) [5]. APA has evolved from "Internet 0" [10], and was originally created to provide a robust Internet working scheme for embedded systems that had many simple parts but an established geometry. Since then, it has been proposed for use in such diverse applications as augmented building infrastructures[11] and motion control for CNC machines [12]

In this particular instantiation of the protocol, every board in the array contains four virtual ports. Each of these boards is assigned an ID that is unique to that board, and contains two sections, called input and output, through which messages can be passed. Each port is also connected to a single neighboring board's port through a two-wire software serial connection. A port can pass a packet in its output section to the input section of the port to which it is connected, or pass a packet from its input section to the output section of a port that is on the same board.

Each packet consists of four parts: a path that contains the sequence of IDs that correspond to the set of ports to be followed, an index that keeps track of the current location within the path, a payload that contains the data that is being transmitted, and a divider that separates the path from the payload. Message passing consists of a port opening the packet it has just received in its input section, reading the ID immediately following the index, replacing ID that was read with the index replacing the blank space left by the index by the ID of the current port, and moving the packet to the output section of the port indicated by the ID that was first read. When the index reaches the divider, this indicates that the packet has reached its destination and is ready to be processed. Additionally, the process of carefully updating the packet path means that the reverse process is already known, allowing for messages to be sent back if need be.

\section{Wireless Network}

Wireless mesh networks have been used for many purposes including military communications, emergency communications, industrial networking of machinery, and many other applications. The ability for each node to communicate with each other wirelessly greatly reduces cost, mass, and complexity in large systems. Unlike in a normal WiFi system, each node will be able to act as a client and a router. Wireless mesh networks used for Internet connectivity has been growing in 
cities to enable larger area wireless coverage. It is also used in developing countries where one node can connect to the Internet, and all the other nodes will be able to have net connectivity though it. A series of nodes could also be used to relay Internet services to remote locations where normal Internet access is hard to reach. There are many applications of wireless mesh networks and bringing them to space will help reduce launch costs, and satellite complexity

As network enabled consumer devices become ubiquitous, advances in network architecture could enable interesting coordinated behavior of many devices. The strategy of composing systems from smaller fungible components has already seen a broad scope of applications, ranging from computing and secure encryption algorithms [13] [14] to overcoming physical limitations for electromagnetic transceiver systems [15]. Recent work on fractionated spacecraft [16] [17] is exploring the application of this strategy to constellations of satellites.

This project takes this a step further, to the components that the satellites are composed of, for the purpose of developing a highly robust spacecraft component system that is either topology agnostic and/or can perform self discovery. A strategy for large scale long duration spacecraft is to build the spacecraft from many fungible components, to allow for dynamic repairability (and reconfigurability, if necessary).

The current system will use the Xbee Zigbee Series 2 wireless modules to integrate into the CubeSat to test the feasibility of the system. After trade studies were conducted to compare with other systems out on the market, we decided that the Zigbee protocol was best for this application due to its ease of implementation.

\section{Power Transfer}

A mesh power network is another technology we are implementing for the project. Because each module is powered by its own battery, the need to keep each module functioning and topped off requires a charging scheme that allows on-demand power.

There are two methods that we are investigating to solve this problem. The first method is a source and sink method of transferring power. In this method, modules will send out a constant signal showing that it is available to transfer power. Neighboring modules that require a charge will detect this signal and open the circuit to accept charge. The other method is a requesting method, where modules that require charge will ask the neighboring modules for power. The block diagram for the charging circuits can be seen in Fig. 2 
 for MRMSS Panel

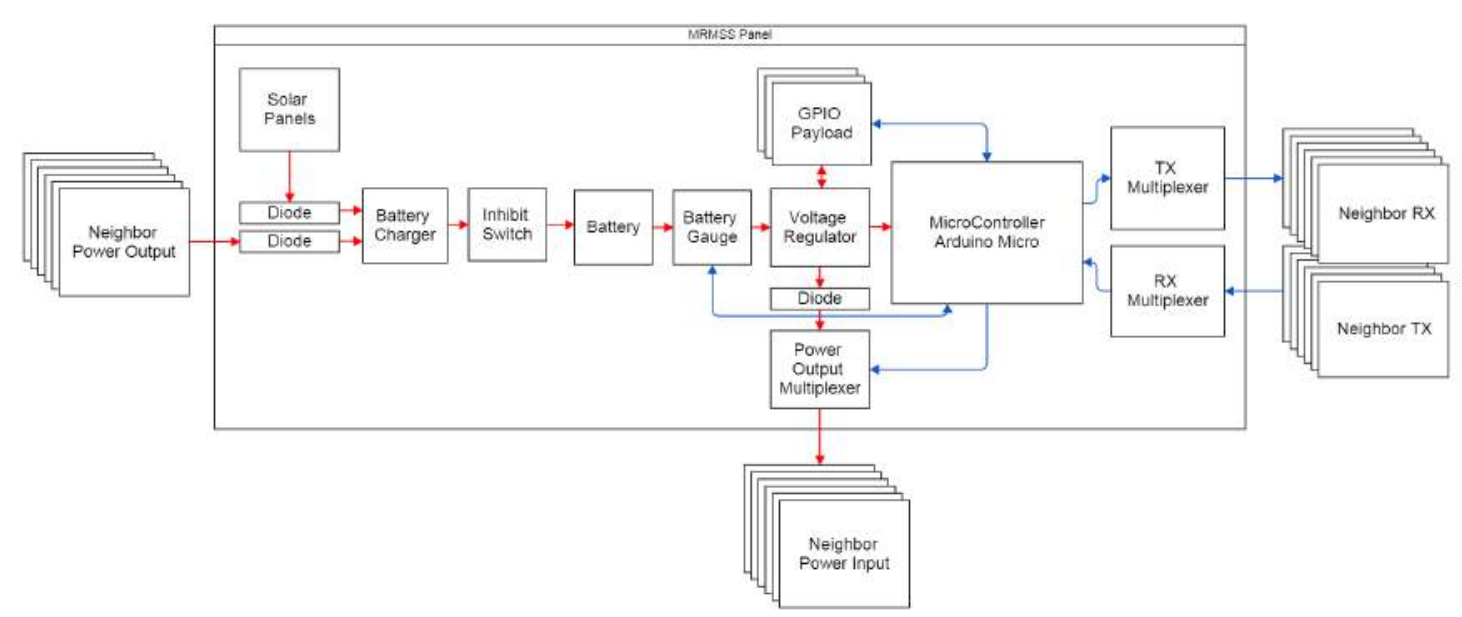

Fig. 2: Power and Data Block Diagram

\section{Payload}

The payload is a demonstration of the modular digital material concept at a much smaller component level. The payload uses Electrical Discharge Machining (EDM) cut pieces, as seen in Fig. 3b assembled to create a capacitor. Electronic digital materials are electrically functional constructions assembled from a small set of discrete parts. With fewer than ten unique parttypes (including conductive, insulating, resistive, and semiconducting) it theorized to be possible to assemble functional three-dimensional micro-electronics. In the module that is incorporated into the MRMSS card system, a capacitor is discretely assembled from insulating and conductive parts. The parts interlock with neighboring ones such that they register to a lattice and have a discrete set of possible positions and orientations. A capacitor is made by placing the conductive parts in an interdigitated finger arrangement, maximizing the area of the capacitor. Electronic digital material structures can be disassembled with the same ease with which they are assembled. This means they don't produce waste and are repairable and reconfigurable. An assembled unit can be seen in Fig. 3a.

This module serves as a test to evaluate the feasibility of using electronic digital materials for space applications. In order to determine the performance of the capacitor during flight, an on board micro-controller measures three parameters: the capacitance of the discretely assembled structure, the resistance across the structure, and the temperature of the environment. These three measures give an indication of how the structure performs with the thermal stresses of space. Because the structure must be preloaded axially in order to maintain electrical conductivity throughout, thermal 


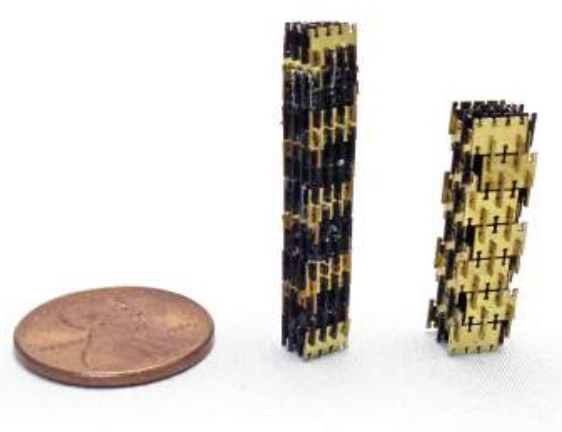

(a) Assembled Discrete Passives

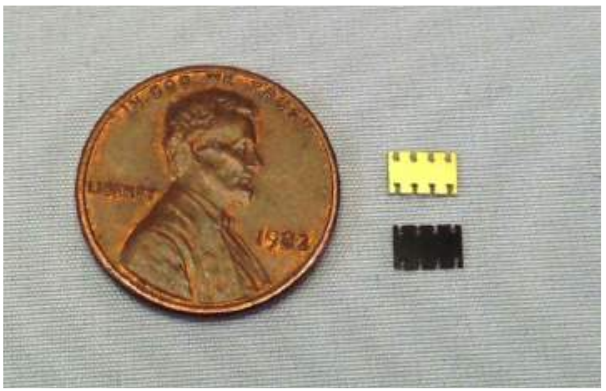

(b) EDM Cut Piece

Fig. 3: Electronic Digital Material [18]

stresses may cause the resistance to change during flight due to axial thermal expansion of the structure. The capacitance, however, should be relatively insensitive to temperature and vibration and therefore serves as our measure of success. Measuring temperature enables us to calibrate for measurement error due to temperature.

The capacitance measurement is made by measuring the RC time constant of the circuit. This enables $100 \mathrm{nF}$ resolution with a roughly 0 to $50 \mathrm{pF}$ range. The resistance measurement uses an instrumentation amplifier to amplify the minute voltage drop across the structure to within a range the 10-bit ADC on the ATtiny44 can process; this enables the measurement of mOhm resistances in a 0 to $500 \mathrm{mOhm}$ range.

\section{Technology Demonstration - Sounding Rocket Experiment}

The opportunity to be a secondary payload on a sounding rocket flight was made available the project through the Sub-Orbital Aerodynamic Re-entry EXperiments (SOAREX) project at NASA Ames Research Center. The SOAREX project is a sounding rocket experiment testing re-entry technologies [19]. The following sections will describe the experiment as well as the communication architecture.

The experiment will launch aboard the Terrier-Peregrine sounding rocket (Flight 12.077). The rocket will have a flight time of about 20 minutes and an approximate maximum altitude of 300 $\mathrm{km}$. For the duration of the flight, the payload will perform three experiments:

1.) Test characteristics of the electronic digital materials.

2.) Verify APA network and perform power charge transfer experiment.

3.) Communicate with the main payload using the 802.15.4 protocol. 


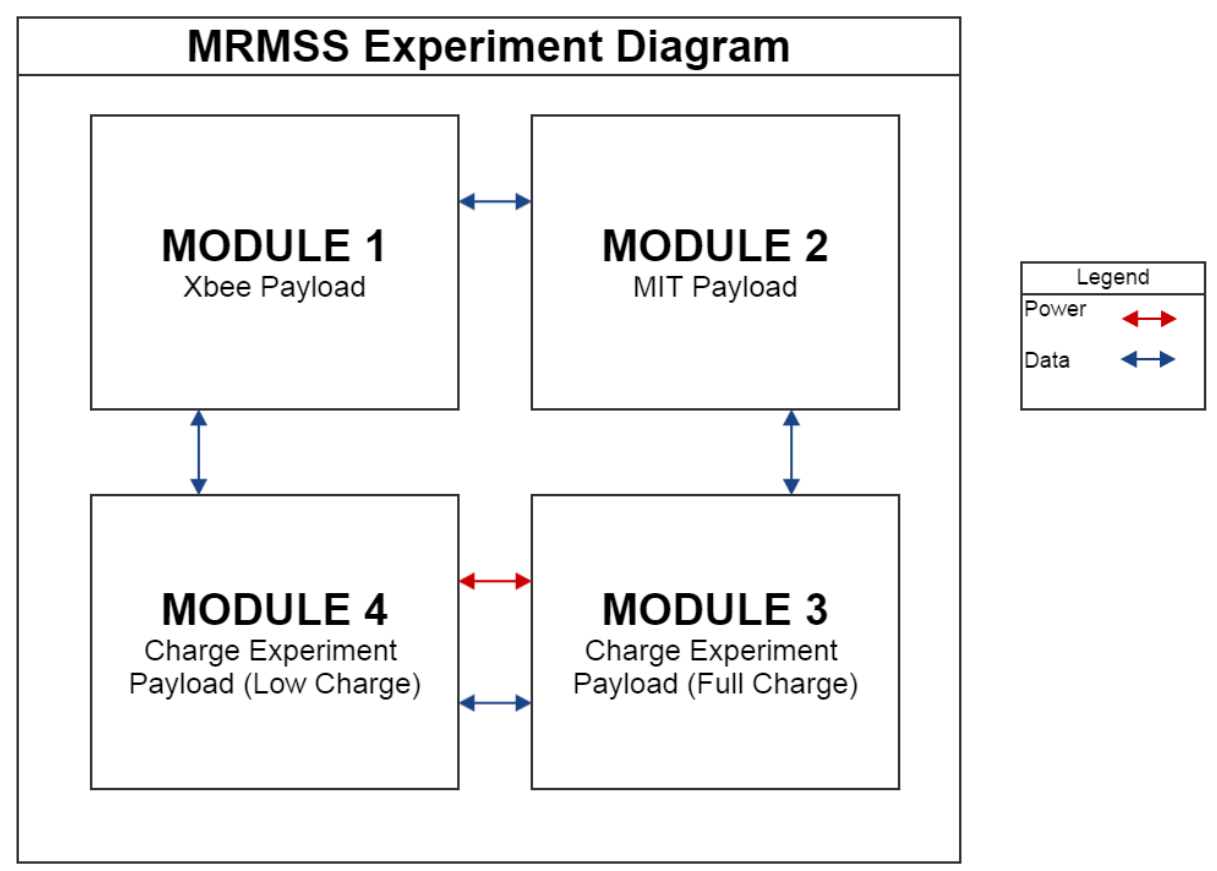

Fig. 4: Experiment Block Diagram

\section{A. Mechanical}

For the sounding rocket flight, the payload consist of 4 of the MRMSS modules attached to the side of the SOAREX spacecraft. Due to volume limitations, these units are only limited to four modules placed in a flat configuration. The four modules are mounted to a flat sheet metal adaptor plate which bolts to the main spacecraft. A prototype unit can be seen on a vibration testing table in Fig. 5.

The unit has been verified through environmental testing, which includes random vibration, thermal vacuum testing, and shock testing.

\section{B. Communication Architecture}

Data from the MRMSS device will be sent to the ground via the SOAREX unit. Communication from the MRMSS payload to the SOAREX unit uses the XBee Series 1 wireless communication module. The reason for using this module is the simplicity of implementing the system. In the future, a Series 2 Zigbee mesh network may be used. It will transfer data to the SOAREX spacecraft using the 802.15.4 protocol. The main spacecraft will then transfer the data using its internal Iridium Modem. The communication concept of operations (ConOps) can be seen in Fig. 6. For this experiment, housekeeping data all four modules as well as the experimental results from the electronic digital material will be sent and used to verify success and performance of the experiment. 


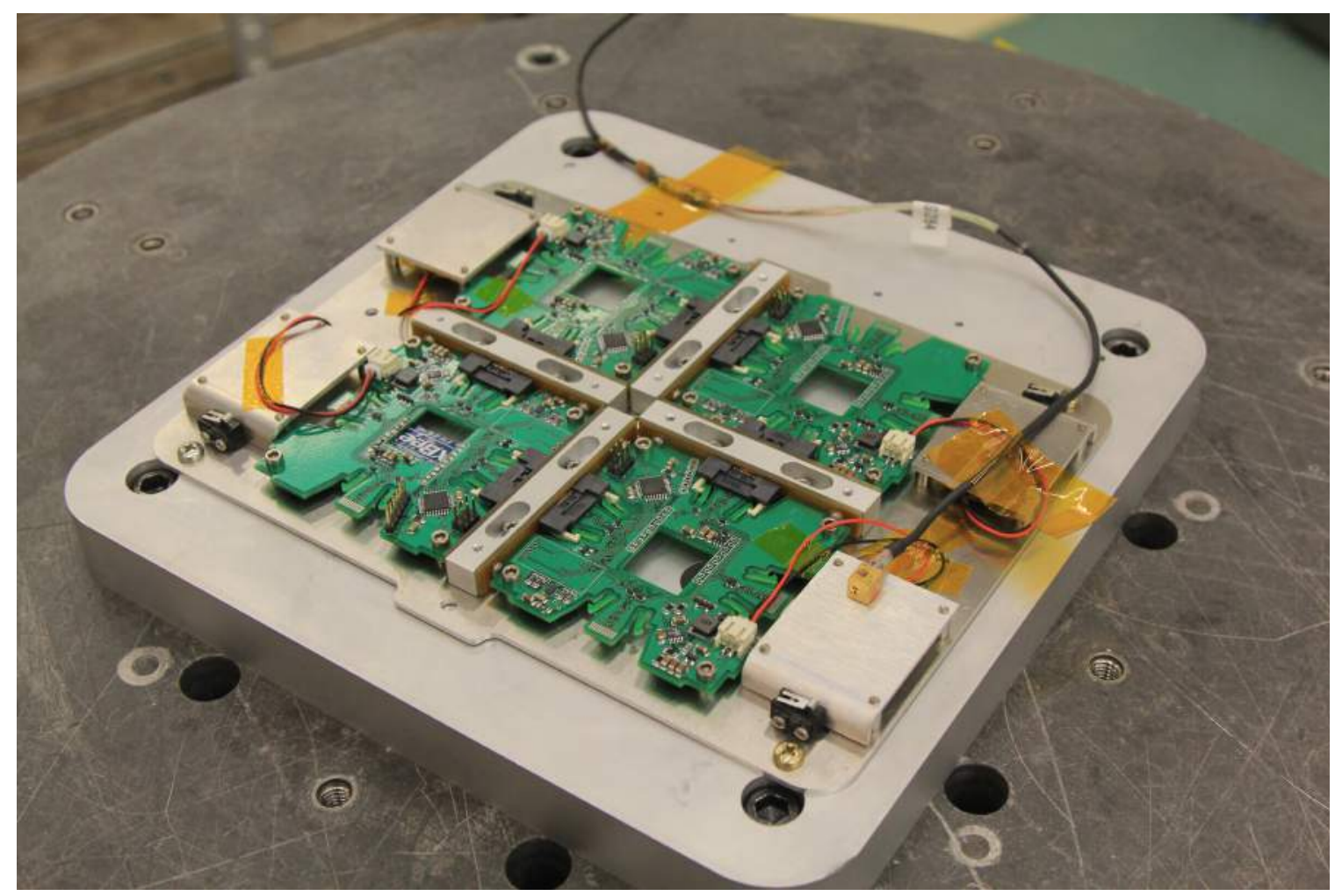

Fig. 5: Experiment Module on Vibration Test Table

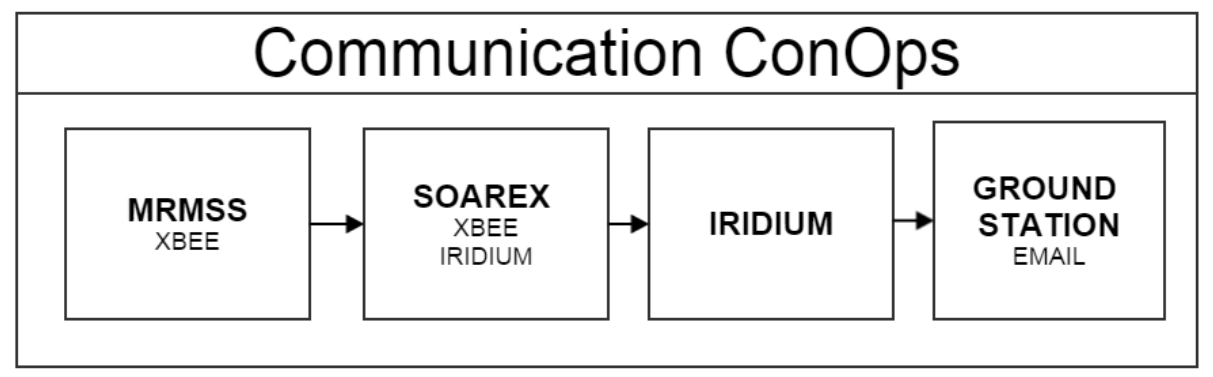

Fig. 6: Communication ConOps

\section{Conclusion}

Digital materials are still in the early stages of development and there are many applications that these technologies can be adapted to. It will be crucial for future spaceflight missions to utilize materials efficiently and re-usability through modular building block based systems concepts can be an important part of this.

This type of concept is a foundation for being able to create space structures that are much larger than what we have been able to create thus far, given launch vehicle constraints. By using discrete modular units, scalable structures for large space stations, telescopes, and other infrastructure could be made possible[20]. At the small scale, digital materials can be used to create mechanical or electronic subsystems and be repurposed once it has completed its job. 
There are three main justifications for the near term application to spacecraft systems. First is the notion that spacecraft built this way would naturally lend themselves to assembly on orbit, which addresses the launch vehicle packing problem as well as reducing the structural requirements imposed by the launch environment. Second is the scalability of core power and data systems, and third is mission profile flexibility, which could be demonstrated in near-term with experiments that include on orbit and on-station procedures as well as packaging and deployment from the station.

Due to the nature of the simple geometry, these units and digital material are particular suited for robotic assembly. Robotic Assembly is one of the key technologies that will be important for building large scale assemblies. A future extension of this project is to look into different ways to assemble these types of digital materials.

\section{Acknowledgments}

The authors of this paper would like to acknowledge NASA's STMD GCD Advanced Manufacturing Technologies project for their support throughout the project, Marcus Murbach and the SOAREX team for sub-orbital flight opportunity. In addition, Gabe Pearhill, Ryan Rivas, and Rick Alena for their guidance on integration of the XBee system. Lastly, we would like to acknowledge MIT Center for Bits and Atoms team for their payload support and foundation research.

\section{References}

[1] Popescu, George, and Neil Gershenfeld. "Digital Materials." MIT CBA[online]. URL: http://fab.cba.mit.edu/classes/MIT/961.09/04.13/DM.draft.pdf[cite 10 Nov. 2014.].

[2] Cheung, Kenneth, and Neil Gershenfeld. "Reversibly Assembled Cellular Composite Materials." Science. 15 Aug. 2013: 1219-221. Print.

[3] "Arduino-ArduinoBoardProMini." Arduino - ArduinoBoardProMini. Web. 22 Dec. 2014. http:// arduino.cc/en/Main/ArduinoBoardProMini.

[4] "Arduino - Introduction." Arduino - Introduction. Web. 22 Dec. 2014. http://arduino.cc/en/Guide/ Introduction.

[5] Oami, Takuma. "FabAcademy 2014 - Takuma Oami." FabAcademy 2014. Web. 22 Dec. 2014. http: //fabacademy.org/archives/2014/students/oami.takuma/networking_and_communications.html.

[6] "Triangular Advanced Solar Cells (TASC) Datasheet." Spectrolab. Web. 22 Dec. 2014. http://www. spectrolab.com/DataSheets/PV/space/TASC_ITJ_datasheet.pdf.

[7] Hartney, Christopher, Elwood Agasid, and Sarah Hovsepian. "Multi-Purpose Avionics Core Element: Using Digital Materials and Advanced Manufacturing to Rapidly Develop Cube Satellite Subsystems 
and Components." ASME 2013 Conference on Smart Materials, Adaptive Structures and Intelligent Systems. American Society of Mechanical Engineers, 2013.

[8] Manchester, Zachary, Mason Peck, and Andrew Filo. "KickSat: A Crowd-Funded Mission to Demonstrate the WorldâĂŹs Smallest Spacecraft." (2013).

[9] "XBee: Connect Devices To The Cloud - Digi International." XBee: Connect Devices To The Cloud Digi International. Web. 23 Dec. 2014. http://www.digi.com/xbee/.

[10] Gershenfeld, Neil, and Danny Cohen. "Internet 0: Interdevice internetworking-End-to-end modulation for embedded networks." Circuits and Devices Magazine, IEEE 22.5 (2006): 48-55.

[11] Gershenfeld, Neil, Stephen Samouhos, and Bruce Nordman. "Intelligent infrastructure for energy efficiency." Science 327.5969 (2010): 1086-1088.

[12] Peek, Nadya. "Motion Control." The Science of Digital Fabrication. MIT Bldg. E14, March 7, 2013.

[13] Gershenfeld, Neil, et al. "Reconfigurable asynchronous logic automata:(RALA)." ACM Sigplan Notices. Vol. 45. No. 1. ACM, 2010.

[14] Standaert, FranÃ ğois-Xavier, et al. "SEA: A scalable encryption algorithm for small embedded applications." Smart Card Research and Advanced Applications. Springer Berlin Heidelberg, 2006. 222-236.

[15] Fenn, Alan J., et al. "The development of phased-array radar technology." Lincoln Laboratory Journal 12.2 (2000): 321-340.

[16] Brown, Owen, Paul Eremenko, and B. Hamilton. "The value proposition for fractionated space architectures." Sciences 99.1 (2002): 2538-2545.

[17] Cockrell, Jim, et al. "EDSN: A Large Swarm of Advanced Yet Very Affordable, COTS-based NanoSats that Enable Multipoint Physics and Open Source Apps." (2012).

[18] Langford, Will. "Electronic Digital Materials." MIT(2014).

[19] "SOAREX (Sub-Orbital Aerodynamic Re-entry EXperiments)." NASA. NASA, 10 Mar. 2005. Web. 23 Dec. 2014. http://www.nasa.gov/centers/ames/research/technology-onepagers/soarex.html.

[20] Watson, Judith, Timothy Collins, and Harold Bush. "A History of Astronaut Construction of Large Space Structures at NASA Langley Research Center." IEEE Xplore. Aerospace Conference Proceedings, 2002. IEEE (Volume:7 ), 1 Jan. 2002. Web. 22 Dec. 2014. 